\title{
Investigation of the caspase-dependent mitochondrial apoptotic pathway in mononuclear cells of patients with systemic lupus erythematosus
}

\author{
Yu-Jih Su ${ }^{1,2}$, Tien-Tsai Cheng ${ }^{2}$, Chung-Jen Chen ${ }^{2}$, Wen-Neng Chang ${ }^{3}$, Nai-Wen Tsai ${ }^{3}$, Chia-Te Kung ${ }^{4}$, \\ Hung-Chen Wang ${ }^{5}$, Wei-Che Lin ${ }^{6}$, Chih-Cheng Huang ${ }^{3}$, Ya-Ting Chang ${ }^{1,3}$, Chih-Min Su ${ }^{1,4}$, Yi-Fang Chiang ${ }^{3}$, \\ Ben-Chung Cheng ${ }^{1,2}$, Yu-Jun Lin ${ }^{1,5}$ and Cheng-Hsien Lu, ${ }^{1,3,7^{*}}$
}

\begin{abstract}
Background: This study aimed to explore the role of apoptosis initiators, caspase-9, caspase-10, mitochondrial anti-viral signaling protein (MAVS), and interferon regulatory factor 7 (pIRF7), in patients with systemic lupus erythematosus (SLE).

Methods: Leukocyte apoptosis was determined by flow cytometry, including annexin V, APO2.7, and 7-aminoactinomycin D (7-AAD) on each subtype of leukocyte in 35 patients with SLE, 15 disease controls, and 17 volunteer normal controls. Levels of caspase-9, caspase-10, MAVS, and pIRF7 in mononuclear cells and the disease activity index (SLEDAI) in the SLE patients were determined. Correlation among intracellular adaptor proteins and caspase levels were calculated.
\end{abstract}

Results: The SLE patients had higher APO2.7 in total leukocyte, lymphocyte, and monocytes, and higher late apoptosis markers in total leukocytes and neutrophils than normal controls (all $p<0.05$ ). Disease activity was positively associated with the APO2.7 of CD19+ cells in SLE, but negatively associated with MAVS and caspase-9 levels (all $p<0.05$ ). Markers of viral infection and anti-virus transcription factors like MDA5, MAVS, and pIRF7 were significantly higher in SLE patients than in disease controls $(p<0.05)$. Caspase-9 and caspase-10 levels positively correlated with MAVS and pIRF7 in SLE patients $(p<0.05)$.

Conclusions: The disease activity of SLE is positively associated with APO2.7 level of CD19+ cells but negatively associated with MAVS and caspase-9 levels, which all point to a mitochondrial pathway.

Keywords: Caspase, Leukocyte apoptosis, Systemic lupus erythematosus, Interferon

\section{Introduction}

Systemic lupus erythematosus (SLE) is a chronic systemic disease affecting mostly women of child-bearing age. It is the prototype of autoimmune diseases because of the variety of its proposed pathogenesis, including apoptosis. A previous study has demonstrated that lymphocyte apoptosis is increased and the removal of apoptotic cells is impaired in SLE. Apoptosis is also higher in active SLE compared to inactive SLE [1].

\footnotetext{
* Correspondence: chlu99@ms44.url.com.tw

'Department of Biological Science, National Sun Yat-Sen University,

Kaohsiung, Taiwan

${ }^{3}$ Departments of Neurology, Kaohsiung Chang Gung Memorial Hospital and

Chang Gung University College of Medicine, Kaohsiung, Taiwan

Full list of author information is available at the end of the article
}

A recent study has demonstrated elevated apoptosis in SLE patients [2] and a correlation between disease activity and the apoptotic marker APO2.7 on CD19+ lymphocytes. Detection of APO2.7 apoptosis indicates that the inner side of the outer membrane of the mitochondria is turned over, which may be initiated by either an extrinsic or intrinsic apoptotic process [3]. The external apoptotic signal is executed through cell surface receptors (e.g. TNFR1, FAS), whereas caspase-10 is activated through proteolytic processes into several active isoforms (e.g. active cleaved form) [3]. Caspase-9 is known either as an initiator caspase of the mitochondrial intrinsic apoptotic pathway or an adaptor of the dependent receptor of apoptosis (Figure 1) [3,4]. 


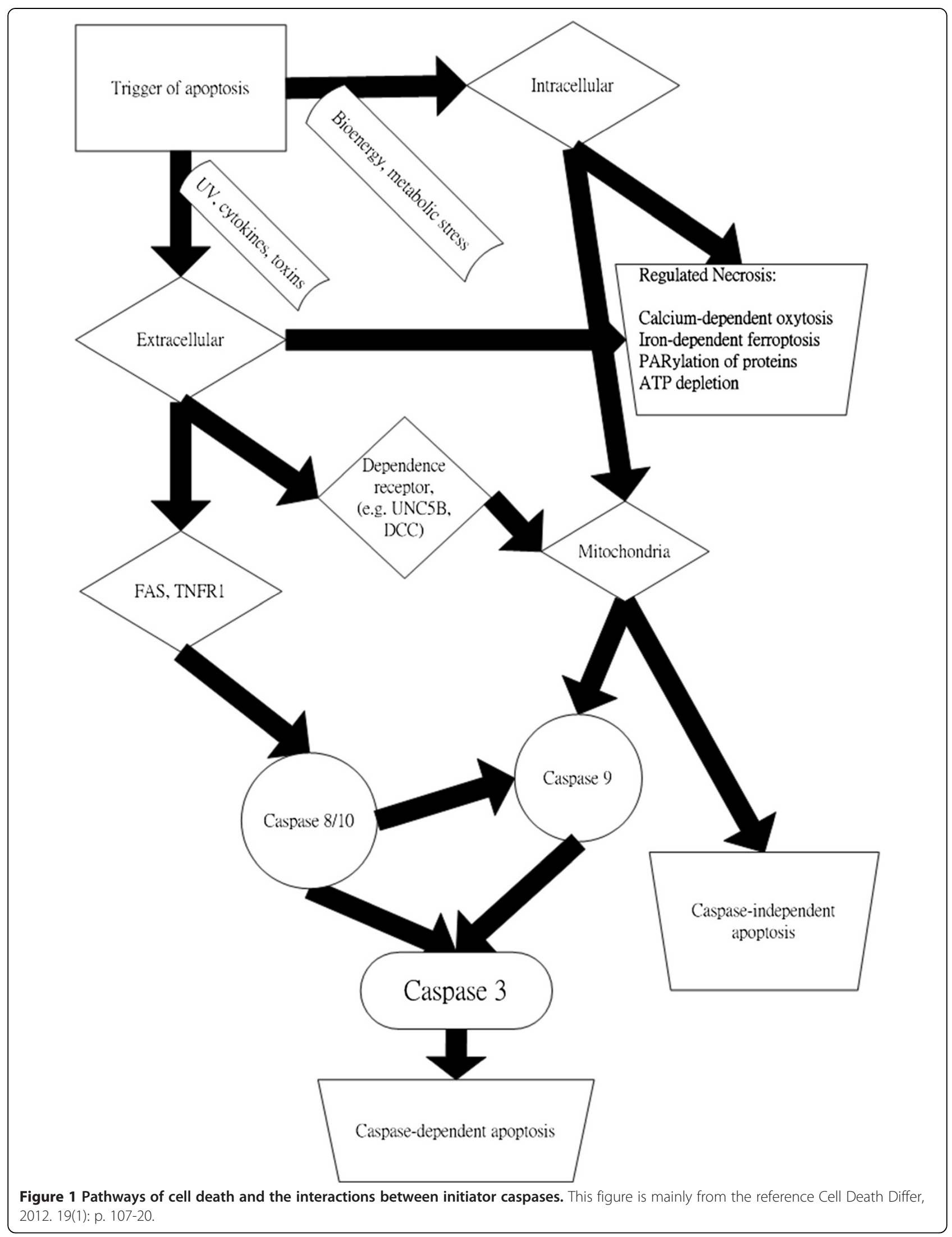


By definition, peripheral blood mononuclear cells (PBMCs) include both lymphocytes and monocytes. In SLE patients, these two lineages of leukocytes are key players of the disease pathogenesis. The apoptotic signature may be started from the bone marrow of SLE patients [5]. Apoptosis and impaired apoptotic clearance are noted in SLE patients and such impaired mechanisms are documented as a pathogenesis of SLE [6]. The major functions of these two leukocyte lines are antigen presentation and the execution of adaptive immunity and interferon production against infection $[7,8]$. Aside from mononuclear cells of leukocytes, viruses have a role in inducing lupus and lupus flare-ups [9-12]. However, with the incorporation of the interferon pathway, the present study focuses on interferon regulatory factor 7 (IRF7) and its related protein in PBMCs, which have not been thoroughly investigated.

The post-viral immune response should induce IRF gene activation [13]. The change in IRF7 phosphorylation levels may be explained by the aberrant activation of the NLRP3 pathway [14], STAT1 pathway [15], or IRF3 [16] downstream of the mitochondrial anti-viral signaling protein (MAVS) due to inflammation. On the other hand, autoimmunity or the cytokine milieu in SLE may also be causes [17-19].

To date, little clinical research has focused specifically on the apoptosis pathway in SLE patients [20]. Because of the possible benefits in choice of appropriate immunosuppressant regimens to reduce the degree of functional morbidity, there is a need to further improve the understanding of the pathogenesis of leukocyte apoptosis. Hence, this study explored the associations among caspases, phosphorylated IRF7 (pIRF7), and APO2.7 apoptosis in patients with SLE.

\section{Patients and methods Study patients}

Thirty-five patients with definitive diagnosis of SLE and followed-up at the Rheumatology Out-patient Clinic for more than six months were prospectively evaluated. The diagnostic criteria for SLE were based on the 1997 revision of the 1982 American College of Rheumatology (ACR) classification criteria for SLE [21], while the clinical assessment of SLE disease activity was based on the SLE disease activity index (SLEDAI) [22]. All SLE patients were regularly followed-up at the clinics for more than six months to ensure that their conditions were stable and that their steroid dose and immune-modifying medication were not changed during the study period. For comparison, 18 age- and sex-matched healthy subjects were enrolled as healthy controls. Fifteen patients with a diagnosis of Sjogren's syndrome, rheumatoid arthritis, vasculitis, or Behcet's disease were also included as disease control patients.
The Institutional Review Committee on Human Research approved the study protocol and all of the participants provided informed consent. Patients were excluded if they had autoimmune diseases other than SLE or if they had fever or any infectious disorder that might affect the WBC count.

\section{Clinical assessments}

All of the subjects underwent complete medical examinations upon enrollment. Complement levels and anti-double strand DNA levels were done regularly and collected at the same time upon enrollment. Biomarkers, including demographic data, complement levels, anti-ribosomal p autoantibody (a-rib p), and anti-double strand DNA autoantibody (a-dsDNA) levels, and disease activity index, were also collected. Since there were no clinical practice guidelines for SLE flare-up, the important decision in immuno-suppressant adjuvant treatment was whether or not to add a cytotoxic or corticosteroid-sparing drug like cyclophosphamide, methotrexate, azathioprine, mycophenolate mofetil, or leflunomide. The daily equivalent dose of steroids and the number of corticosteroid-sparing drugs used by SLE and disease control patients were recorded.

\section{Blood sampling and assessment of leukocyte apoptosis}

Blood samples were collected by venipuncture of forearm veins of the SLE patients and controls.

\section{Flow cytometry assay for detecting apoptosis}

All flow cytometry assays were performed within one hour after blood extraction to ensure that the results were as close as possible to an in vivo situation. Fixed amounts of blood were diluted 1:5 with PBS and $100 \mu \mathrm{L}$ was stained with $10 \mu \mathrm{L}$ of each of the following: fluorescence conjugated monoclonal antibodies against CD45-phycoerythrin (PE)-Cy5 (clone J33), CD61-fluorescein isothiocyanate (FITC; clone SZ21), and APO 2.7-PE (clone 2.7A6A3; Immunotech, Marseille, France), and titrated at saturating concentrations. The CD45-PE-Cy5 antibody reacted with the CD45 family of trans-membrane glycoproteins, which were expressed on the surface of all human leukocytes and were pan-leukocyte markers. The CD61-FITC antibody was a pan-platelet marker that reacted with the GPIIb/IIIa complex (fibrinogen receptor). The APO 2.7-PE antibody reacted with a $38-\mathrm{kDa}$ mitochondrial membrane protein (7A6 antigen), which was detectable in non-permeabilized cells in the late apoptotic state [23].

Annexin V staining, relevant to early apoptosis, produced similar results but was rejected for questionable reliability under fixation conditions, with formaldehyde clearly biasing the staining results. Mouse immunoglobulin G-PE was a control for non-specific staining, which did not differ from the APO2.7-PE signal on platelets, such that each subject was used as its own control without changing the sample tube. After 30 minutes of incubation 
in the dark at room temperature, the stained samples were diluted with $0.5 \mathrm{ml}$ of FACSFlow (Becton Dickinson, San Jose, CA).

Flow cytometry analysis was performed immediately after staining using an Epics XL flow cytometer (Beckman Coulter, Fullerton, Calif) and CellQuest software. Five thousand CD45-PE-Cy5+ cells per sample were acquired in a combined forward and side scatters and deep-red FL4 fluorescence (CD45-PE-Cy5) leukocyte gate. Another 5000 CD61-FITC + cells per sample were acquired in a combined forward and side scatters and green FL1 fluorescence (CD61-FITC) platelet gate to define a negative control threshold for the measurement of apoptosis. Each subject was his/her own control.

Membrane phosphatidyl-serine was detected by annexin$\mathrm{V}$ using a commercially available kit (Boehringer Mannheim, Indianapolis, Ind). The PBS-washed leucocytes were incubated with annexin V-FITC and 7-amino-actinomycin D (7-AAD) for $15 \mathrm{~min}$ at room temperature according to the manufacturer's guidelines. Samples were transferred to $5 \mathrm{~mL}$ polypropylene tubes, diluted with $900 \mu \mathrm{L}$ Hanks' balanced salt solution, and placed on ice before analysis by flow cytometry. The samples were analyzed using an Epics XL flow cytometer (Beckman Coulter, Fullerton, CA) and CellQuest software. Fifteen thousand events were counted per sample. Low-fluorescence debris was gated-out of the analysis. Leukocyte subtypes were identified according to their CD45 expression intensity and divided into neutrophils, monocytes, and lymphocytes.

Annexin V-FITC staining was identified in fluorescent-1 and 7-AAD staining in fluorescent-4. The cells were identified as follows: early apoptotic cells if they were positive for marker annexin V-FITC but negative for 7-AAD; late apoptotic cells if they were positive for annexin V-FITC and 7-AAD; dead cells if they were negative for annexin V-FITC but positive for 7-AAD; and viable cells if they were negative for annexin V-FITC and 7-AAD.

Fixed amounts of blood were diluted 1:5 with PBS, while $100 \mu \mathrm{L}$ was stained with $10 \mu \mathrm{L}$ of each of the following: fluorescence conjugated monoclonal antibodies against CD4-phycoerythrin (PE)-Cy5, CD19-fluorescein isothiocyanate (FITC), and CD8- phycoerythrin (PE). After each of the above staining, further staining was done with annexin V-FITC, 7-amino-actinomycin D (7-AAD), or APO 2.7-PE (clone 2.7A6A3; Immunotech, Marseille, France), with titration at saturating concentrations. The samples were then transferred to $5 \mathrm{~mL}$ polypropylene tubes, diluted with $900 \mu \mathrm{L}$ Hanks' balanced salt solution, and placed on ice before flow cytometry.

The samples were analyzed using an Epics XL flow cytometer (Beckman Coulter, Fullerton, Calif) and CellQuest software. Fifteen thousand events were counted per sample. Lymphocyte subtypes were identified according to their surface antigen (i.e., CD4+, CD8+, or CD19+) expression intensity. A database coordinator was responsible for monitoring all data collection and entry.

\section{Western blot analysis}

Peripheral blood mononuclear cell (PBMC) intracellular protein levels of caspase-9, caspase-9c, caspase-10, caspase10-c, and pIRF7 were detected by Western blot method. The PBMCs were separated by Ficoll-Paque (GE Healthcare Bio-Sciences AB, Stockholm, Sweden) density gradient centrifugation. The PBMC protein was extracted with RIPA Buffer containing $1 \mathrm{mmol} / \mathrm{L}$ protease inhibitor and $1 \mathrm{mmol} / \mathrm{L}$ phosphatase inhibitor. Proteins with sample buffer were then boiled for $10 \mathrm{~min}$ and aliquots of the supernatants (40 $\mathrm{\mu g} / \mathrm{lane})$ were fractionated by electrophoresis in $10 \%$ SDS acrylamide gel, electro-transferred to PVDF membranes, and blocked with $5 \%$ non-fat powdered milk in TBS-T (20 mmol/L Tris-base [pH 7.6], $137 \mathrm{mmol} / \mathrm{L} \mathrm{NaCl}, 0.5 \%$ Tween-20).

The membranes were then incubated with commercially available rabbit polyclonal antibodies recognizing caspase-9 (Cell signaling, \#9501), phospho-IRF-7 (Cell signaling, \#5184), rat polyclonal antibodies recognizing caspase-10 (Biolegend, \#645202) at $4^{\circ} \mathrm{C}$ overnight. Following extensive washing with TBS-T, the blots were incubated for 60 min with secondary antibodies. After brief rinsing with TBS-T, immuno-reactive protein bands were visualized by a chemiluminescence-based procedure using the ECL detection kit (Thermo, SuperSignal West Femto Maximum Sensitivity Substrate) according to the manufacturer's instructions. The signal was quantified densitometrically by Quantity One 1-D analysis software (Bio-Rad USA, Life Science Research, Hercules, CA).

Activated caspase- 9 was demonstrated by cleaved caspase-9 (active caspase-9, caspase-9c, $37 \mathrm{kd}$ ) from original caspase-9 (caspase-9, 47 kd) [18]. Activated caspase-10 was demonstrated by cleaved caspase-10 (active caspase-10, caspase-10c, $43 \mathrm{kd}$ ) from original caspase-10 (caspase-10, $59 \mathrm{kd)}$ [19].

\section{Determination of intracellular protein concentration by enzyme linked-immuno-sorbent assay (ELISA)}

Intracellular protein levels and serum levels of MDA5 were detected by commercial ELISA kit. Aliquots of the supernatants of protein $(40 \mu \mathrm{g})$ were tested using a commercial ELISA kit to determine the concentration of intracellular MDA5 (APOtech, \#APO-54 N-035, Epalinges, Switzerland). A commercial ELISA kit was used to determine the concentration of serum MDA5 (Cusabio, CSB-EL011017HU, China).

\section{Statistical analysis}

Data were expressed as mean \pm SD or median (inter-quartile range). Categorical variables were compared using Chi-square test or Fisher's exact test, as appropriate. 
Continuous variables (i.e., surface markers of leukocytes and intracellular protein levels) were square root transformed to improve normality, and then compared between two groups using Student's t-test and among three groups using one-way analysis of variance (ANOVA). Correlation analysis was used to explore the relationship between the mean steroid dosages and mean SLEDAI score, and variables such as surface markers of leukocytes, and intracellular protein levels. Statistical significance was set at $p<0.05$. All statistical calculations were performed using the SAS software package, version 9.1 (2002, SAS Statistical Institute, North Carolina).

Table 1 Demographic data of SLE patients and healthy controls

\begin{tabular}{|c|c|c|c|c|}
\hline & Healthy controls $(n=18)$ & SLE patients $(n=35)$ & Disease control $(n=15)$ & $p$ value \\
\hline Age $(y)($ mean $\pm S D)$ & $43.78 \pm 10.90$ & $40.24 \pm 11.78$ & $47.53 \pm 11.06$ & 0.17 \\
\hline Male/female & $5 / 13$ & $3 / 32$ & $1 / 13$ & 0.92 \\
\hline \multicolumn{5}{|l|}{ Peripheral blood studies } \\
\hline Leukocytes (×1000/ml) & $5.85 \pm 2.15$ & $6.35 \pm 2.61$ & $7.92 \pm 3.11$ & 0.12 \\
\hline$\%$ granulocyte & $61.60 \pm 12.76$ & $67.89 \pm 12.63$ & $72.36 \pm 16.25$ & 0.25 \\
\hline \% lymphocyte & $31.01 \pm 10.85$ & $23.65 \pm 10.87$ & $20.46 \pm 14.77$ & 0.18 \\
\hline$\%$ monocyte & $5.63 \pm 1.74$ & $6.14 \pm 2.71$ & $4.63 \pm 2.10$ & 0.48 \\
\hline Hemoglobin (mg/dL) & $13.32 \pm 1.77$ & $11.73 \pm 1.73$ & $12.52 \pm 1.58$ & $<0.01^{*}$ \\
\hline Platelet counts (×10000/ml) & $23.19 \pm 6.82$ & $22.19 \pm 8.91$ & $23.05 \pm 8.60$ & 0.93 \\
\hline c3 level (median, IQR) & ND & $91.0(61.4,107.5)$ & ND & \\
\hline c4 Level (median, IQR) & ND & $19.30(9.2,24)$. & ND & \\
\hline a-dsDNA (median, IQR) & ND & $13.4(1.78,67)$ & ND & \\
\hline a-rib p (median, IQR) & ND & $0.10(0.1,18)$ & ND & \\
\hline SLEDAI score ( median, IQR) & ND & $6(4,12)$ & ND & \\
\hline \multicolumn{5}{|l|}{ Medication used during at blood test } \\
\hline Equivalent prednisolone dose per day (mg) & & $0(0,8.75)$ & $0(0,8.75)$ & 0.90 \\
\hline Immune modulators except steroid (number) & & $1(1,1)$ & $1(1,1.25)$ & 0.48 \\
\hline \multicolumn{5}{|l|}{ Leukocyte Apoptosis by flow cytometry } \\
\hline \multicolumn{5}{|l|}{ Leukocyte apoptosis (\%) } \\
\hline Annexin V (\%) & $10.7(8.9,13.8)$ & $11.9(8.6,17.1)$ & $12.1(9.3,20.6)$ & 0.41 \\
\hline APO2.7 (\%) & $0.7(0.5,0.9)$ & $1.3(0.9,2.0)$ & $1.17(0.9,2.1)$ & $<0.01^{*}$ \\
\hline Annexin V + 7AAD (\%) & $3.34(2.85,4.78)$ & $6.34(3.7,9.7)$ & $7.06(5.16,13.95)$ & $<0.01^{*}$ \\
\hline \multicolumn{5}{|l|}{ Neutrophil apoptosis (\%) } \\
\hline Annexin V (\%) & $14.5(10.0,16.7)$ & $19.4(11.5,29.8)$ & $18.9(14.4,28.5)$ & 0.08 \\
\hline APO2.7 (\%) & $0.51(0.39,0.62)$ & $0.57(0.41,1.6)$ & $0.82(0.35,1.39)$ & 0.18 \\
\hline Annexin V + 7AAD (\%) & $3.7(2.1,7.6)$ & $8.6(2.7,19.2)$ & $15.94(10.9,31.4)$ & $<0.01^{*}$ \\
\hline \multicolumn{5}{|l|}{ Lymphocyte apoptosis (\%) } \\
\hline Annexin V (\%) & $4.85(4.01,7.26)$ & $6.28(4.18,9.52)$ & $6.97(4.00,12.47)$ & 0.16 \\
\hline APO2.7 (\%) & $0.34(0.22,0.45)$ & $0.67(0.4,0.8)$ & $0.79(0.60,1.01)$ & $<0.01^{*}$ \\
\hline Annexin V + 7AAD (\%) & $1.76(1.17,2.40)$ & $2.12(1.31,2.87)$ & $2.20(1.22,3.54)$ & 0.49 \\
\hline \multicolumn{5}{|l|}{ Monocyte apoptosis (\%) } \\
\hline Annexin V (\%) & $16.7(13.0,21.8)$ & $16.4(10.9,22.1)$ & $18.2(14.0,23.9)$ & 0.65 \\
\hline APO2.7 (\%) & $1.7(1.05,2.44)$ & $2.8(1.9,5.2)$ & $2.3(1.3,4.6)$ & $0.02^{*}$ \\
\hline Annexin V + 7AAD (\%) & $5.5(4.2,6.6)$ & $8.0(4.3,14.4)$ & $8.2(4.5,17.0)$ & 0.07 \\
\hline
\end{tabular}

Abbreviations: SLE, systemic lupus erythematosus; IQR, inter-quartile range.

Data presented with median (IQR) or mean \pm SD.

Continuous variables among three groups were compared using one-way ANOVA (analysis of variance) between healthy group, SLE, and diseases control.

Comparison between the three groups with non-normalized distributed parameters was calculated using the Kruskal-Wallis test, while post-hoc analysis was by Mann-Whitney U test.

${ }^{*} p<0.05$. 


\section{Results}

Baseline characteristics of the study patients

The baseline characteristics, laboratory data, and apoptosis markers of the SLE patients, diseases controls, and healthy controls were listed in Table 1 . The 15 diseases control patients included ten with Sjogren's syndrome, two with rheumatoid arthritis, two with vasculitis, and one with Behcet's disease. The clinical symptoms of the 35 SLE patients included neurologic involvement in 15, musculo-skeletal involvement in 10, hematologic involvement in seven, renal involvement in five, cardio-respiratory involvement in three, and muco-cutaneous involvement in three. Ten SLE patients had more than one organ involvement. There was no significant difference in age and sex among the three groups. The mean dosages of steroids and the number of immune-modifying medication were similar between the SLE and disease control groups $(p>0.05)$ (Table 1).

Leukocyte apoptosis in patients with SLE and the controls The laboratory data and percentage of leukocyte apoptosis among the three groups were also listed in Table 1. Except for hemoglobin level, all of the cell counts were not significantly different. Nonetheless, the SLE patients had higher apoptosis markers, including higher APO2.7 $(p<0.0001)$ and late apoptosis markers $(p=0.0019)$ in leukocytes. There were higher late apoptosis markers among neutrophils $(p=0.0479)$, higher APO2.7 apoptosis markers among monocytes $(p=0.0018)$, and higher APO2.7 apoptosis markers among lymphocytes $(p=0.0004)$ (Table 1).

\section{Correlation between clinical severity and percentage of monocytes and lymphocyte apoptosis}

Correlation analysis was used to test the influence of leukocyte apoptosis on SLEDAI disease activity. The statistical results (correlation coefficient, $p$ value) revealed that only the percentage of APO2.7 on CD19+ cells was positively associated with disease activity $(r=0.59$, $p=0.01$ ) (Table 2). The other subsets of leukocyte apoptosis were not correlated with SLEDAI disease activity. Further exploring the relationship between percentage of APO2.7 of CD19+ cell apoptosis and levels of autoantibodies revealed no correlation. Specifically, percentage of APO2.7 of CD19+ cell apoptosis did not correlate with the level of anti-Ro52 $(\mathrm{r}=0.35, p=0.39)$, antiRo60 ( $\mathrm{r}=0.29, p=0.48)$, anti-La $(\mathrm{r}=0.23, p=0.34)$, anti-RNP ( $\mathrm{r}=0.01, p=0.99)$, anti-Sm $(\mathrm{r}=0.41, p=0.11)$, anti-dsDNA $(\mathrm{r}=-0.28, p=0.22), \mathrm{C} 3(\mathrm{r}=-0.16, p=0.49)$, or $\mathrm{C} 4$ ( $\mathrm{r}=0.01, p=0.98)$. Lastly, there was no significant relationship between the percentage of each apoptosis marker (e.g., Annexin V, APO2.7, annexin V + 7AAD) and steroid dosage in the SLE patients (all $p>0.05$ ) (Table 2).
Table 2 Correlation analysis between leukocyte apoptosis markers and disease activity and steroid dosage in patients with systemic lupus erythematosus

\begin{tabular}{|c|c|c|c|c|}
\hline & \multicolumn{2}{|c|}{$\begin{array}{l}\text { SLE diseases } \\
\text { activity }\end{array}$} & \multicolumn{2}{|c|}{$\begin{array}{l}\text { Steroid } \\
\text { dosage }\end{array}$} \\
\hline & $\mathbf{r}$ & $\mathbf{p}$ & $\mathbf{r}$ & $\mathbf{p}$ \\
\hline CD4+ cell Annexin V (\%) & 0.02 & 0.93 & 0.01 & 0.96 \\
\hline CD4+ cell Annexin V + 7AAD (\%) & -0.09 & 0.70 & -0.33 & 0.12 \\
\hline CD8+ cell Annexin V (\%) & -0.30 & 0.19 & -0.17 & 0.45 \\
\hline CD8+ cell Annexin V + 7AAD (\%) & -0.06 & 0.79 & -0.19 & 0.39 \\
\hline CD19+ cell Annexin V (\%) & 0.17 & 0.46 & -0.19 & 0.39 \\
\hline CD19+ cell Annexin V + 7AAD (\%) & 0.12 & 0.61 & -0.15 & 0.50 \\
\hline Monocyte Annexin V (\%) & 0.01 & 0.97 & -0.11 & 0.54 \\
\hline Monocyte Annexin V + 7AAD (\%) & 0.04 & 0.85 & -0.14 & 0.46 \\
\hline APO2.7 CD4+ cell (\%) & 0.17 & 0.51 & -0.29 & 0.20 \\
\hline APO2.7 CD8+ cell (\%) & 0.32 & 0.19 & -0.12 & 0.61 \\
\hline APO2.7 CD19+ cell (\%) & 0.59 & $0.01^{*}$ & 0.17 & 0.46 \\
\hline APO2.7 monocyte (\%) & 0.05 & 0.77 & -0.17 & 0.35 \\
\hline
\end{tabular}

Abbreviations: $r$, correlation coefficient; $p, p$ value; SLEDAI, Systemic lupus erythematosus disease activity index 2000 . ${ }^{*} p<0.05$.

\section{Correlation between each intracellular protein level and} disease activity in SLE patients

The intracellular proteins, including active cleaved caspase 10c (43 kD), caspase 9c (37 kD), active MAVS (57 kD), and pIRF7 (65 kD) were determined by Western blot method (Figure 2a), while MDA5 levels were determined by ELISA (Figure 2b). The correlation between each intracellular protein, including MDA5, active cleaved caspase-9, active cleaved caspase-10, pIRF7, and active MAVS revealed that active cleaved caspase- 9 and active MAVS level both negatively correlated with SLEDAI $(p=0.01$ and $p<0.05$, respectively) (Table 3).

Active cleaved caspase- 9 positively correlated with active cleaved caspase-10 $(p<0.01)$. Both the active cleaved caspase- 9 and caspase- 10 positively correlated with pIRF7 $(p<0.01)$. Despite the absence of correlation between MDA5 level and other molecules, activated MAVS positively correlated with the active cleaved caspase-9, caspase-10, and pIRF7 (all $p<0.01$ ).

\section{Correlation between original and cleaved active caspase- 9 and -10 in SLE}

In PBMCs, caspase-10 was positively associated with caspase-10c $(p<0.001)$ (Figure 3a). Caspase-9 and caspase-10 positively correlated with each other $(p<0.001)$ (Figure 3b). Caspase- 9 positively associated with active cleaved casepase-9c $(p<0.001)$ (Figure 3c). 


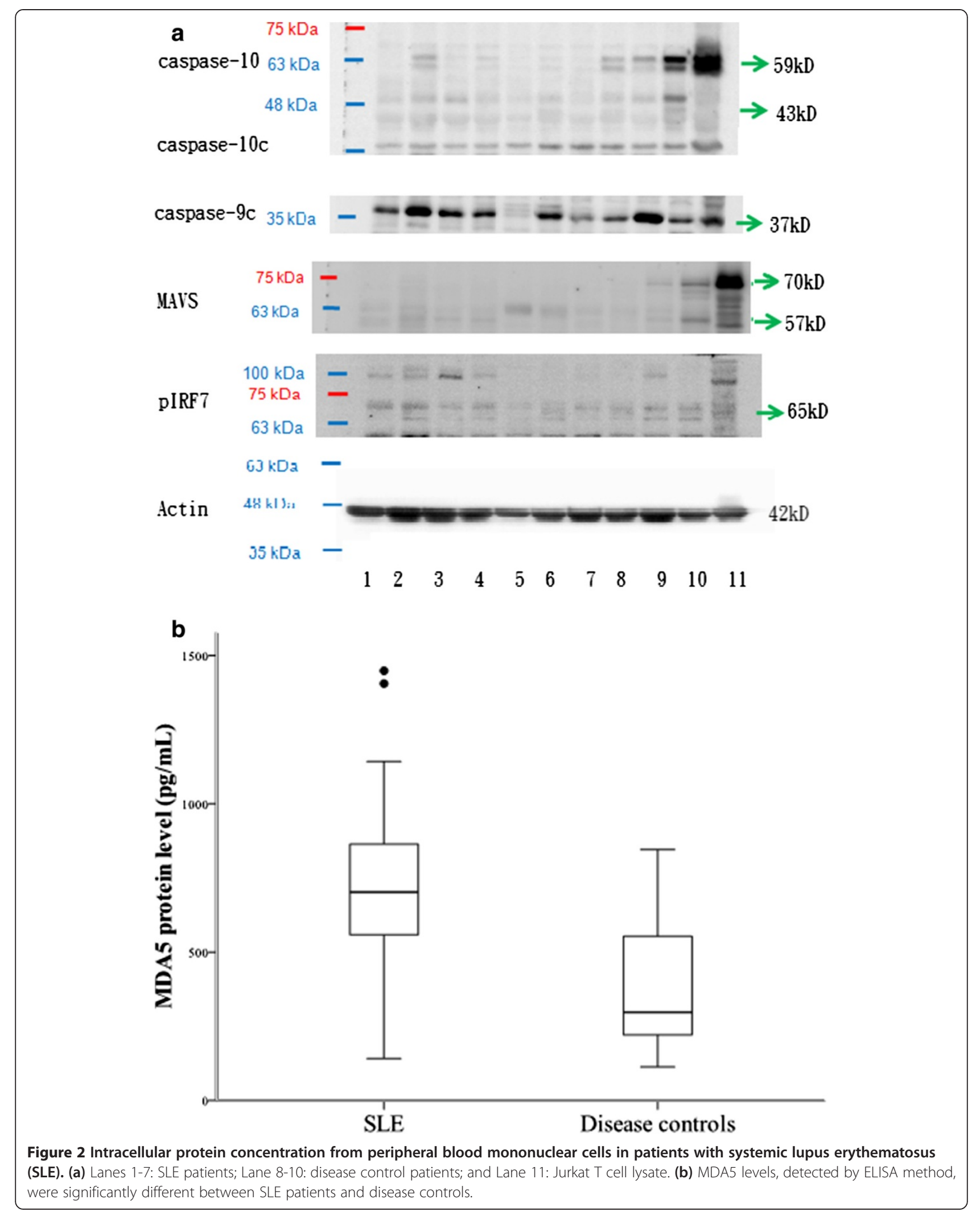


Table 3 Correlations analysis between activated caspase-9, activated caspase-10, pIRF7, virus infection markers, and systemic lupus erythematosus disease activity

\begin{tabular}{|c|c|c|c|c|c|c|c|}
\hline & & SLEDAI & activated caspase 9 & activated caspase 10 & pIRF7 & activated MAVS & MDA5 \\
\hline \multirow[t]{2}{*}{ SLEDAI } & $r$ & 1.00 & $-0.47^{*}$ & -0.31 & -0.31 & $-0.36^{*}$ & -0.03 \\
\hline & $p$ & $x$ & $0.01^{*}$ & 0.13 & 0.09 & $<0.05^{*}$ & 0.90 \\
\hline \multirow[t]{2}{*}{ activated caspase 9} & r & & 1.00 & $0.81^{*}$ & $0.83^{*}$ & $0.88^{*}$ & 0.39 \\
\hline & $p$ & & $x$ & $<0.01^{*}$ & $<0.01^{*}$ & $<0.01^{*}$ & 0.06 \\
\hline \multirow[t]{2}{*}{ activated caspase 10} & $r$ & & & 1.00 & $0.91^{*}$ & $0.85^{*}$ & 0.23 \\
\hline & $\mathrm{p}$ & & & $x$ & $<0.01^{*}$ & $<0.01^{*}$ & 0.33 \\
\hline \multirow[t]{2}{*}{ pIRF7 } & r & & & & 1.00 & $0.84^{*}$ & 0.14 \\
\hline & $p$ & & & & $x$ & $<0.01^{*}$ & 0.51 \\
\hline \multirow[t]{2}{*}{ activated MAVS } & $r$ & & & & & 1.00 & 0.27 \\
\hline & $p$ & & & & & $x$ & 0.21 \\
\hline \multirow[t]{2}{*}{ MDA5 } & $r$ & & & & & & 1.00 \\
\hline & $p$ & & & & & & $x$ \\
\hline
\end{tabular}

Abbreviations: $r$, correlation coefficient; $p, p$ value; SLEDAl, Systemic lupus erythematosus disease activity index 2000; pIRF7, phosphorylated interferon regulato factor 7; MAVS, mitochondrial antiviral signaling protein; MDA5, Melanoma Differentiation-Associated protein 5. ${ }^{*} p<0.05$.

\section{Correlation between disease activity and MAVS protein level in SLE}

The negative coefficient between MAVS and SLE disease activity (Table 3) was enhanced if patients with lower MDA5 levels (cut-off value, $366 \mathrm{pg} / \mathrm{mL}$, mean of disease controls) were removed (Figure 4). The $p$ value changed from 0.04955 to 0.01123 , indicating that SLE was a heterogeneous group and could be divided into at least two subgroups according to MDA5 levels. Disease activity negatively correlated with MAVS in the high MDA5 level SLE patients $(p<0.05)$ but not in the low MDA5 level SLE patients $(p=0.67)$ (data not shown).

\section{Discussion}

The present study has several major findings. First, there are comparable apoptosis markers between SLE and disease control patients, even though only neutrophil late apoptosis is statistically significantly higher in disease controls than in SLE patients $(p<0.05)$ (Table 1). Second, SLE disease activity is positively associated with CD19+ cell APO 2.7 marker level (Table 2), but negatively associated with MAVS and caspase-9 levels (Table 3) $(p<0.05)$. Third, although most of the surface markers of apoptosis are not significantly different between SLE and disease controls, the levels of intracellular molecules are significantly different $(p<0.05)$ (Table 4$)$. Fifth, markers of viral infection and anti-virus transcription factors, including MDA5, MAVS, and pIRF7, are significantly higher in SLE patients than in disease controls $(p<0.05)$ (Table 4). Sixth, caspase- 9 and caspase-10 levels are positively correlated with anti-virus factors like MAVS and pIRF7 $(p<0.05)$ (Table 3), but not MDA5 levels. Lastly, SLE disease activity is more negatively associated with MAVS among patients with high MDA5 levels (Figure 3).
This present study confirms that pronounced apoptosis in SLE and disease activity is positively associated with APO2.7 of CD19+ cells [2]. Disease activity is also negatively associated with caspase- 9 and MAVS in PBMCs (Table 3). These all point to a mitochondrial pathway. The explanation may be that when disease activity increases, the PBMCs are activated and anti-apoptotic molecules accumulate on the mitochondrial membrane [24-27], which suppress caspase-9. Moreover, another article mentions that the expression of caspase 9 is decreased in female SLE patients [20]. Active cleaved caspase- 9 may come from any lineage of PBMCs, such as activation-inducedcell-death of $\gamma \delta$-T cell, which depends on caspase-9 [28], or through pathways not dependent on caspase in other CD4+ T cells [29].

During disease flare-up, CD19+ cells are prone to producing more autoantibodies with mitochondrial activation, which may lead to APO2.7-related apoptosis. However, the detailed apoptosis mechanism remains unknown. On the other hand, the reason that caspase-10 is not correlated with disease activity may be that there are too many confounding factors affecting the extrinsic apoptosis pathway, like TNF- $\alpha$ [30] and other stress signaling [31].

The negative correlation between disease activity and MAVS is contrary to a previous clinical observation [9]. Thus, the role of MDA5 in this correlation has been investigated by determining the levels of MDA5 among SLE and disease control patients. A previous study comparing MDA5 levels between SLE and healthy controls [32] shows the MDA5 is significantly higher in SLE than in healthy patients. In the present study, MDA5 levels, as well as the anti-virus signaling proteins MAVS and pIRF7, are significantly higher in SLE than in disease control patients (Table 4). This indicates that SLE patients are 

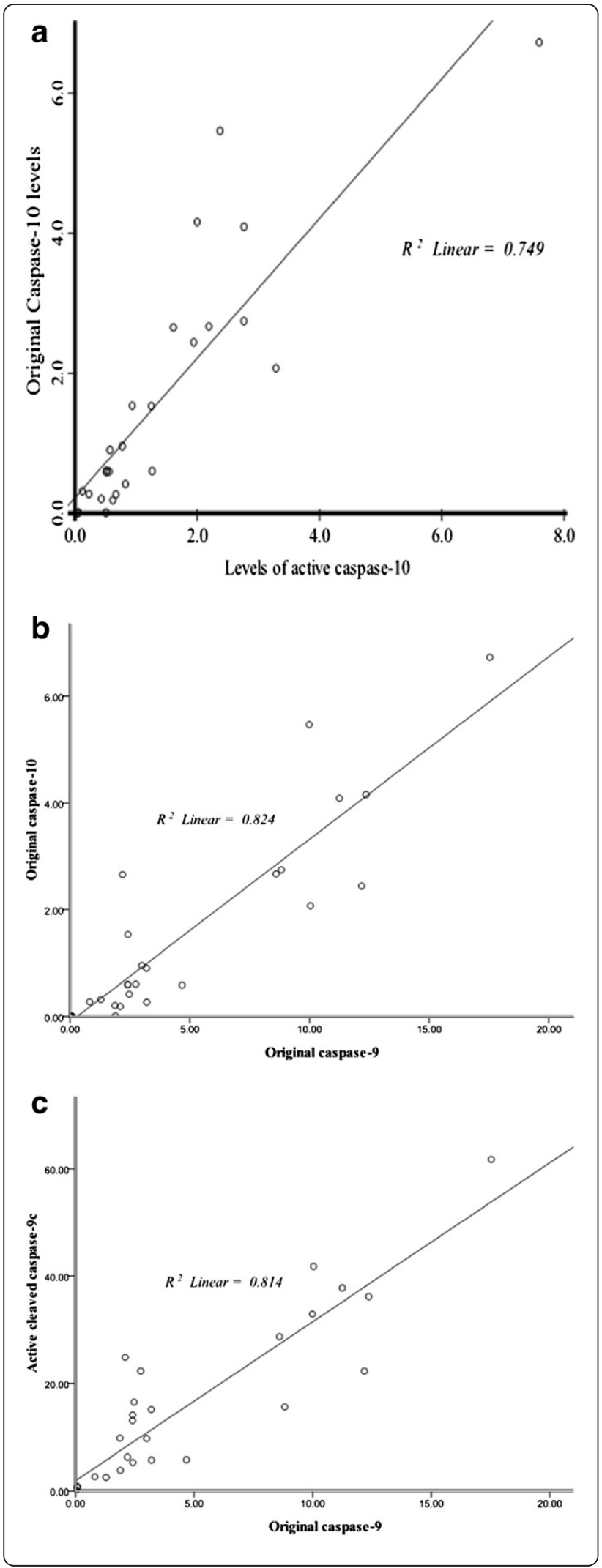

Figure 3 Correlations between original and cleaved caspase- 9 and caspase-10 in systemic lupus erythematosus patients. (a) In PBMCs, original caspase-10 was positively associated with active cleaved casepase-10c $(p<0.05)$. (b) Original caspase-10 was positively associated with original caspase-9 $(p<0.05)$. (c) Original caspase-9 was positively associated with active cleaved casepase-9 $(p<0.05)$. Abbreviations: $r$, correlation coefficient.

more vulnerable to recurrent viral infection than disease controls.

Furthermore, using the mean MDA5 level from disease controls as reference, disease activity is negatively correlated to MAVS in SLE patients with high MDA5 levels $(p<0.05)$, but not in those with low MDA5 levels $(p=0.67)$ (Figure 4$)$. This indicates that MAVS is only negatively associated with disease activity in a subset of SLE patients. Further studies to determine the specific role of MDA5 and MAVS in SLE are needed.

The three major IRFs (i.e., IRF3, IRF5, and IRF7) are downstream of mitochondrial signaling to promote interferon-stimulated gene expression [33,34]. According to a previous study, SLE is an interferon-associated disease. Thus, this study focused on interferon regulating factors, particularly on IRF7 because of a previous study by Becker et al. [31], wherein IRF7 is the only one significantly different between healthy and SLE CD19+ cells. In the current study, SLE CD19+ cells are associated with disease activity in SLE patients.

Caspase-9 is mainly an intracellular initiator caspase and is related to extrinsic apoptosis from dependent receptors [3]. In the current study, caspase-9c is positively associated with MAVS and pIRF7 (Table 3). It will be interesting to elucidate the relationship between viral infection and caspase-9 activation, since virus-related cellular apoptosis has been demonstrated in some HIV infected patients [35], while caspase-9 activation is noted in chronic hepatitis C viral infection [36]. Caspase-9c and MAVS are both negatively associated with disease activity in this study (Table 3), which may hint that activation-induced-celldeath through mitochondrial pathway is different from the virus-related apoptosis and/or activation pathway in SLE patients. Virus could lead to IRF7 down-regulation [37] or activation [13,38,39]. Virus-related activation and activation-induced apoptosis of mononuclear cells in SLE through the mitochondrial pathway may exist but warrants further study.

There are several positive correlations between caspase10c and MAVS, as well as caspase-10c and pIRF7 (Table 3). These indicate that caspase-10, like caspase-9, may be another major initiator of the caspase pathway in PBMCs in SLE. Caspase-10c is produced mainly after caspase-10 is activated and cleaved. Its molecular weight is around $43 \mathrm{kD}$ [40]. Both the original caspase-10 (59 kD) and caspase-10c (43 kD) have the large protease subunit p20, 

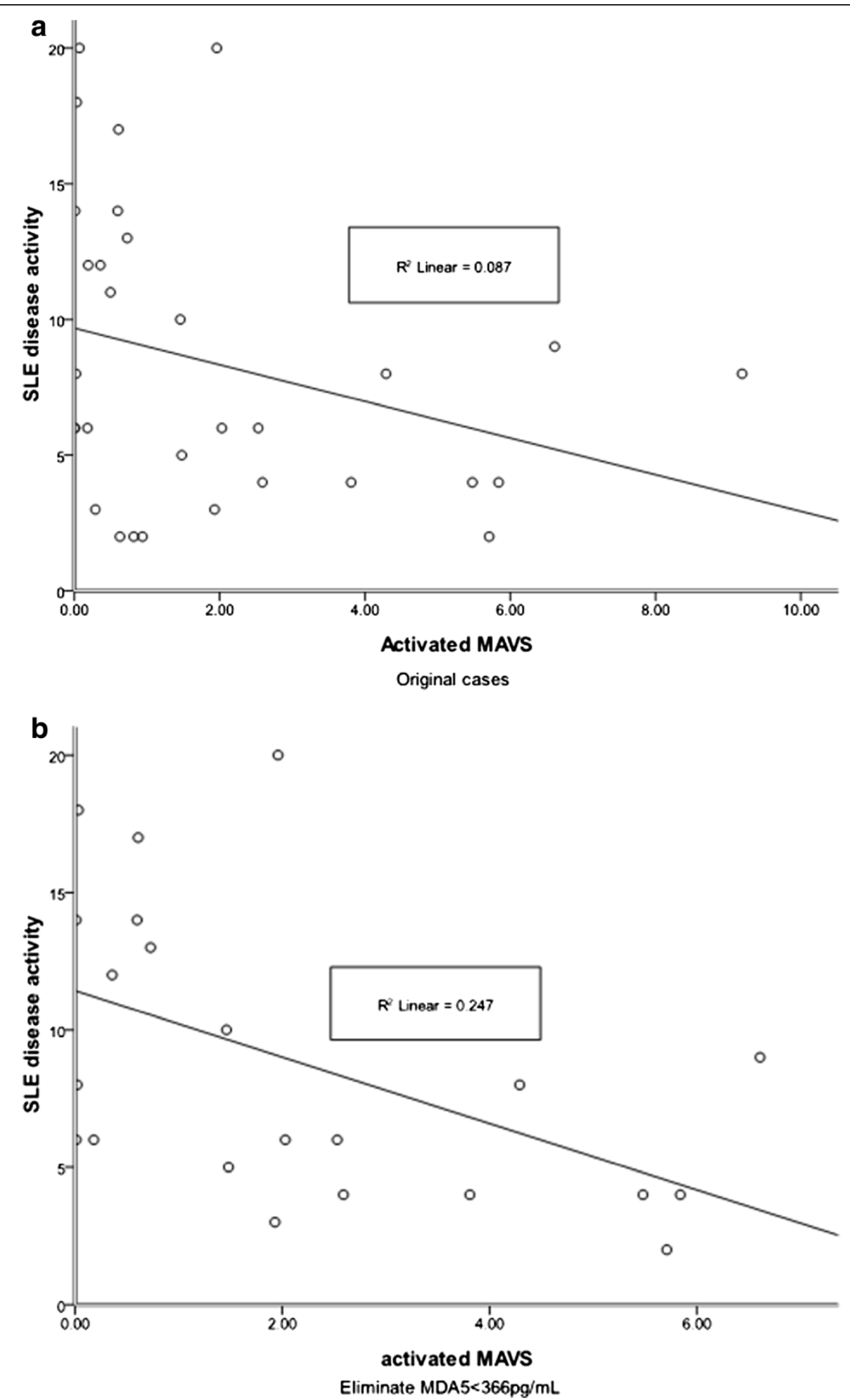

Figure 4 Correlation between MAVS and SLEDAI. (a) Origin correlation between MAVS and SLEDAI. (b) Correlation between MAVS and SLEDAI after eliminating the low-MDA5 sub-group. 
Table 4 Comparison of intracellular molecules between patients with systemic lupus erythematosus and disease controls

\begin{tabular}{|c|c|c|c|}
\hline & SLE & Disease controls & \\
\hline & $\mathrm{n}=31$ & $n=15$ & $p$ value \\
\hline \multicolumn{4}{|l|}{ Intracellular protein levels } \\
\hline MDA5 (pg/mL) & $701.53(543.45,885.96)$ & $296.74(216.50,567.40)$ & $<0.01^{*}$ \\
\hline Activated caspase 9 & $9.74(0.81,22.34)$ & $2.20(0.26,3.58)$ & 0.06 \\
\hline Activated caspase 10 & $0.77(0.51,2.09)$ & $0.15(0.04,0.24)$ & $<0.01^{*}$ \\
\hline pIRF7 & $0.10(0.04,0.30)$ & $0.03(0.01,0.04)$ & $<0.01^{*}$ \\
\hline Activated MAVS & $0.82(0.19,2.59)$ & $0.24(0.11,0.42)$ & $0.02^{*}$ \\
\hline
\end{tabular}

Data presented with median, IQR; IQR, inter-quartile range.

The levels of pIRF7, activated caspase 9, caspase 10, and MAVS from Western blot method were normalized with actin levels.

Abbreviation: pIRF7, phosphorylated interferon regulator factor 7; MAVS, mitochondrial anti-viral signaling protein.

${ }^{*} p<0.05$.

recognized by the secondary antibody in Western blotting. Both molecules are functionally active [40].

Nonetheless, this study has several limitations. First, theoretically, it would have been better if PBMCs were divided into different cell lineages. In this study, as in other studies, PBMCs are used as a surrogate rather than lymphocytes only because of the technical difficulty to differentiate lymphocytes from monocytes using flow cytometry study $[41,42]$. Because lymphocytes account for the majority of PMBCs, the results are not much different from the true state. Second, this is a crosssectional observational study. Theoretically, immunosuppressant drugs can affect leukocyte activity and autoantibody levels even if SLE patients are in the convalescent stage. Lastly, the case number in this study is small. Large-scale prospective and longitudinal studies are needed to evaluate the prognostic contribution of leukocyte apoptosis on clinical outcome.

\section{Ethical approval}

The study was approved by Chang Gung Memorial Hospital's Institutional Review Committee on Human Research.

\section{Competing interests}

The authors declare that they have no competing interests.

\section{Authors' contributions}

YJS participated in the design of the study and drafted the manuscript. CJC, TTC, WNC, CTK, HCW, WCL, CCH, YTC, CMS, YFC, BCC, and YJL participated in the sequence alignment and clinical evaluation of patients. NWT performed the statistical analysis. CHL conceived the study, participated in its design and coordination, and helped draft the manuscript. All of the authors read and approved the final manuscript.

\section{Authors' information}

Yu-Jih Su, Ya-Ting Chang, Chih-Min Su, Ben-Chung Cheng, Yu-Jun Lin, and Cheng-Hsien Lu are from the Department of Biological Science, National Sun Yat-Sen University, Kaohsiung, Taiwan.

Yu-Jih Su, Tien-Tsai Cheng, Chung-Jen Chen and Ben-Chung Cheng are also physicians from the Department of Internal Medicine; Wen-Neng Chang, Cheng-Hsien Lu, Nai-Wen Tsai, Chih-Cheng Huang and Ya-Ting Chang are from the Department of Neurology; Chia-Te Kung and Chih-Min Su are physicians from the Department of Emergency Medicine; Hung-Chen Wang and Yu-Jun Lin are surgeons from the Department of Neurosurgery; and Wei-Che Lin is a radiologist from the Department of Radiology of Chang
Gung Memorial Hospital-Kaohsiung Medical Center, Chang Gung University College of Medicine, Kaohsiung, Taiwan.

\section{Acknowledgement}

This work was supported by grants from Chang Gung Memorial HospitalKaohsiung Medical Center and the Ministry of Science and Technology (CMRPG8B1281 and NHRI-EX101-10142EI). The authors wish to thank Dr. Gene Alzona Nisperos for reviewing the manuscript for English language considerations.

\section{Author details}

${ }^{1}$ Department of Biological Science, National Sun Yat-Sen University, Kaohsiung, Taiwan. ${ }^{2}$ Departments of Internal Medicine, Kaohsiung Chang Gung Memorial Hospital and Chang Gung University College of Medicine, Kaohsiung, Taiwan. ${ }^{3}$ Departments of Neurology, Kaohsiung Chang Gung Memorial Hospital and Chang Gung University College of Medicine, Kaohsiung, Taiwan. ${ }^{4}$ Departments of Emergency Medicine, Kaohsiung Chang Gung Memorial Hospital and Chang Gung University College of Medicine, Kaohsiung, Taiwan. ${ }^{5}$ Departments of Neurosurgery, and Radiology, Kaohsiung Chang Gung Memorial Hospital and Chang Gung University College of Medicine, Kaohsiung, Taiwan. 'Departments of Radiology, Kaohsiung Chang Gung Memorial Hospital and Chang Gung University College of Medicine, Kaohsiung, Taiwan. ${ }^{7}$ Department of Neurology, Chang Gung Memorial Hospital, 123, Ta Pei Road, Niao Sung Hsiang, Kaohsiung, Taiwan.

Received: 7 July 2014 Accepted: 21 October 2014

Published online: 06 November 2014

\section{References}

1. Jin O, Sun LY, Zhou KX, Zhang XS, Feng XB, Mok MY, Lau CS: Lymphocyte apoptosis and macrophage function: correlation with disease activity in systemic lupus erythematosus. Clin Rheumatol 2005, 24(2):107-110.

2. Su YJ, Cheng TT, Chen CJ, Chiu WC, Hsu CY, Chang WN, Tsai NW, Kung CT, Wang HC, Lin WC, Chih-Cheng H, Ya-Ting C, Chih-Min S, Yi-Fang C, BenChung C, Yu-Jun L, Cheng-Hsien L: The association among leukocyte apoptosis, autoantibodies and disease severity in systemic lupus erythematosus. J Trans/ Med 2013, 11(1):261.

3. Galluzzi L, Vitale I, Abrams JM, Alnemri ES, Baehrecke EH, Blagosklonny MV, Dawson TM, Dawson VL, El-Deiry WS, Fulda S, Gottlieb E, Green DR, Hengartner MO, Kepp O, Knight RA, Kumar S, Lipton SA, Lu X, Madeo F, Malorni W, Mehlen P, Nuñez G, Peter ME, Piacentini M, Rubinsztein DC, Shi Y, Simon H-U, Vandenabeele P, White E, Yuan J, et al: Molecular definitions of cell death subroutines: recommendations of the Nomenclature Committee on Cell Death 2012. Cell Death Differ 2012, 19(1):107-120.

4. Vanden Berghe T, Linkermann A, Jouan-Lanhouet S, Walczak H, Vandenabeele P: Regulated necrosis: the expanding network of non-apoptotic cell death pathways. Nat Rev Mol Cell Biol 2014, 15(2):135-147.

5. Park J, Moon S, Lee J, Lee D, Jung K, Song Y, Lee E: Bone marrow analysis of immune cells and apoptosis in patients with systemic lupus erythematosus. Lupus 2014, 23(10):975-985. 
6. Navratil JS, Ahearn JM: Apoptosis, clearance mechanisms, and the development of systemic lupus erythematosus. Curr Rheumatol Rep 2001, 3(3):191-198.

7. Gong X, Zhou J, Zhu W, Liu N, Li J, Li L, Jin Y, Duan Z: Excessive proinflammatory cytokine and chemokine responses of human monocyte-derived macrophages to enterovirus 71 infection. BMC Infect Dis 2012, 12:224

8. Ekchariyawat $\mathrm{P}$, Thitithanyanont A, Sirisinha S, Utaisincharoen P: Apoptosis induced by avian $\mathrm{H} 5 \mathrm{~N} 1$ virus in human monocyte-derived macrophages involves TRAIL-inducing caspase-10 activation. Innate Immun 2012 , 18(3):390-397

9. Su BY, Su CY, Yu SF, Chen CJ: Incidental discovery of high systemic lupus erythematosus disease activity associated with cytomegalovirus viral activity. Med Microbiol Immunol 2007, 196(3):165-170.

10. Berner BR, Tary-Lehmann M, Yonkers NL, Askari AD, Lehmann PV, Anthony DD: Phenotypic and functional analysis of EBV-specific memory CD8 cells in SLE. Cell Immunol 2005, 235(1):29-38.

11. Dreyfus DH: Role of T cells in EBV-infected systemic lupus erythematosus patients. J Immunol 2005, 175(6):3460. author reply 3461.

12. Gross AJ, Hochberg D, Rand WM, Thorley-Lawson DA: EBV and systemic lupus erythematosus: a new perspective. J Immunol 2005, 174(11):6599-6607.

13. Honda K, Yanai H, Negishi H, Asagiri M, Sato M, Mizutani T, Shimada N, Ohba Y, Takaoka A, Yoshida N, Taniguchi T: IRF-7 is the master regulator of type-l interferon-dependent immune responses. Nature 2005 434(7034):772-777.

14. Park S, Juliana C, Hong S, Datta P, Hwang I, Fernandes-Alnemri T, Yu JW, Alnemri ES: The mitochondrial antiviral protein MAVS associates with NLRP3 and regulates its inflammasome activity. J Immunol 2013, 191(8):4358-4366

15. Nandakumar R, Finsterbusch K, Lipps C, Neumann B, Grashoff M, Nair S, Hochnadel I, Lienenklaus S, Wappler I, Steinmann E, Hauser H, Pietschmann T, Kröger A: Hepatitis C Virus Replication in Mouse Cells Is Restricted by IFN-Dependent and -Independent Mechanisms. Gastroenterology 2013, 145(6):1414-1423. e1411.

16. Di Paolo NC, Doronin K, Baldwin LK, Papayannopoulou T, Shayakhmetov DM: The transcription factor IRF3 triggers "defensive suicide" necrosis in response to viral and bacterial pathogens. Cell reports 2013, 3(6):1840-1846.

17. Fu Q, Zhao J, Qian X, Wong JL, Kaufman KM, Yu CY, Mok MY, Harley JB, Guthridge JM, Song YW, Cho SK, Bae SC, Grossman JM, Hahn BH, Arnett FC, Shen N, Tsao BP: Association of a functional IRF7 variant with systemic lupus erythematosus. Arthritis Rheum 2011, 63(3):749-754

18. Wang F, Zou YF, Sun GP: Association between functional IRF7 variant and systemic lupus erythematosus may need more critical examination: comment on the article by Fu et al. Arthritis Rheum 2011, 63(10):3177-3178. author reply 3178-3179.

19. Uematsu S, Sato S, Yamamoto M, Hirotani $T$, Kato H, Takeshita $F_{\text {, }}$ Matsuda M, Coban C, Ishii KJ, Kawai T, Takeuchi O, Akira S: Interleukin-1 receptor-associated kinase- 1 plays an essential role for Toll-like receptor (TLR)7- and TLR9-mediated interferon-\{alpha\} induction. J Exp Med 2005, 201(6):915-923.

20. Rastin M, Mahmoudi M, Hatef M, Sahebari M, Tabasi N, Haghmorad D, Nosratabadi R, Zamani S, Khazaee M, Masoudian M: T lymphocyte apoptosis in systemic lupus erythematosus patients. Iran J Basic Med Sci 2013, 16(8):936-941.

21. Smith EL, Shmerling RH: The American College of Rheumatology criteria for the classification of systemic lupus erythematosus: strengths, weaknesses, and opportunities for improvement. Lupus 1999, 8(8):586-595.

22. Bombardier C, Gladman DD, Urowitz MB, Caron D, Chang CH: Derivation of the SLEDAl. A disease activity index for lupus patients. The Committee on Prognosis Studies in SLE. Arthritis Rheum 1992, 35(6):630-640.

23. Koester SK, Roth P, Mikulka WR, Schlossman SF, Zhang C, Bolton WE: Monitoring early cellular responses in apoptosis is aided by the mitochondrial membrane protein-specific monoclonal antibody APO2.7. Cytometry 1997, 29(4):306-312.
24. Liphaus BL, Kiss MH: The role of apoptosis proteins and complement components in the etiopathogenesis of systemic lupus erythematosus. Clinics (Sao Paulo) 2010, 65(3):327-333.

25. Graninger WB, Steiner CW, Graninger MT, Aringer M, Smolen JS: Cytokine regulation of apoptosis and $\mathrm{BCl}-2$ expression in lymphocytes of patients with systemic lupus erythematosus. Cell Death Differ 2000, 7(10):966-972

26. Xie M, Wang Z, Liu X, Qiang O, Liu G: [Preliminary study on apoptosis and expression of apoptosis-related genes in peripheral blood lymphocytes of patients with systemic lupus erythematosus]. Hua Xi Yi Ke Da Xue Xue Bao 1999, 30(2):192-195.

27. Seki M, Ushiyama C, Seta N, Abe K, Fukazawa T, Asakawa J, Takasaki Y, Hashimoto H: Apoptosis of lymphocytes induced by glucocorticoids and relationship to therapeutic efficacy in patients with systemic lupus erythematosus. Arthritis Rheum 1998, 41(5):823-830.

28. Atre N, Thomas L, Mistry R, Pathak K, Chiplunkar S: Role of nitric oxide in heat shock protein induced apoptosis of gammadeltaT cells. Int $J$ Cancer 2006, 119(6):1368-1376.

29. Bolton DL, Hahn BI, Park EA, Lehnhoff LL, Hornung F, Lenardo MJ: Death of CD4(+) T-cell lines caused by human immunodeficiency virus type 1 does not depend on caspases or apoptosis. J Virol 2002, 76(10):5094-5107.

30. Ng PW, Porter AG, Janicke RU: Molecular cloning and characterization of two novel pro-apoptotic isoforms of caspase-10. J Biol Chem 1999, 274(15):10301-10308.

31. Rikhof B, Corn PG, El-Deiry WS: Caspase 10 levels are increased following DNA damage in a p53-dependent manner. Cancer Biol Ther 2003, 2(6):707-712.

32. Becker AM, Dao KH, Han BK, Kornu R, Lakhanpal S, Mobley AB, Li QZ Lian Y, Wu T, Reimold AM, Olsen NJ, Karp DR, Chowdhury FZ, Farrar JD, Satterthwaite AB, Mohan C, Lipsky PE, Wakeland EK, Davis LS: SLE peripheral blood $B$ cell, $T$ cell and myeloid cell transcriptomes display unique profiles and each subset contributes to the interferon signature. PLoS One 2013, 8(6):e67003.

33. Lazear HM, Lancaster A, Wilkins C, Suthar MS, Huang A, Vick SC, Clepper L, Thackray L, Brassil MM, Virgin HW, Nikolich-Zugich J, Moses AV, Gale M Jr, Früh K, Diamond MS: IRF-3, IRF-5, and IRF-7 coordinately regulate the type I IFN response in myeloid dendritic cells downstream of MAVS signaling. PLOS Pathog 2013, 9(1):e1003118.

34. Takaki H, Honda K, Atarashi K, Kobayashi F, Ebihara T, Oshiumi H, Matsumoto M, Shingai M, Seya T: MAVS-dependent IRF3/7 bypass of interferon beta-induction restricts the response to measles infection in CD150Tg mouse bone marrow-derived dendritic cells. Mol Immunol 2014, 57(2):100-110.

35. Yue FY, Kovacs CM, Dimayuga RC, Gu XX, Parks P, Kaul R, Ostrowski MA: Preferential apoptosis of HIV-1-specific CD4+ T cells. J Immunol 2005, 174(4):2196-2204

36. Albertoni G, Arnoni CP, Latini FR, Andrade SS, Araujo PR, Rodrigues FK, Rozenchan PB, Mendes-Correa MC, Leite OH, Schor N, Girão MJ, Barreto JA: Altered of apoptotic markers of both extrinsic and intrinsic pathways induced by hepatitis $C$ virus infection in peripheral blood mononuclear cells. Virol J 2012, 9:314.

37. Wang J, Yang B, Hu Y, Zheng Y, Zhou H, Wang Y, Ma Y, Mao K, Yang $L$, Lin G, Ji $Y$, Wu $X$, Sun B: Negative regulation of $\mathrm{Nmi}$ on virus-triggered type I IFN production by targeting IRF7. J Immunol 2013, 191(6):3393-3399.

38. Honda K, Ohba Y, Yanai H, Negishi H, Mizutani T, Takaoka A, Taya C, Taniguchi T: Spatiotemporal regulation of MyD88-IRF-7 signalling for robust type-I interferon induction. Nature 2005, 434(7036):1035-1040.

39. Oganesyan G, Saha SK, Guo B, He JQ, Shahangian A, Zarnegar B, Perry A, Cheng G: Critical role of TRAF3 in the Toll-like receptordependent and -independent antiviral response. Nature 2006, 439(7073):208-211.

40. Mologni L, Ponzanelli I, Bresciani F, Sardiello G, Bergamaschi D, Gianni M, Reichert U, Rambaldi A, Terao M, Garattini E: The novel synthetic retinoid 6-[3-adamantyl-4-hydroxyphenyl]-2-naphthalene carboxylic acid (CD437) causes apoptosis in acute promyelocytic leukemia cells through rapid activation of caspases. Blood 1999, 93(3):1045-1061. 
41. Cacciapaglia F, Spadaccio C, Gregorj C, Margiotta D, Coccia R, De Marco F, Chello M, Picardi A, Amoroso A, Afeltra A: Apoptosis and autoimmunity induced by clodronate in systemic lupus erythematosus mononuclear circulating cells. Int I Immunopathol Pharmacol 2010, 23(2):535-542.

42. Zhi-Chun L, Qiao-Ling Z, Zhi-Qin L, Xiao-Zhao L, Xiao-xia Z, Rong T: Tumor necrosis factor-like weak inducer of apoptosis (TWEAK) mediates p38 mitogen-activated protein kinase activation and signal transduction in peripheral blood mononuclear cells from patients with lupus nephritis. Inflammation 2012, 35(3):935-943.

doi:10.1186/s12967-014-0303-1

Cite this article as: Su et al.: Investigation of the caspase-dependent mitochondrial apoptotic pathway in mononuclear cells of patients with systemic lupus erythematosus. Journal of Translational Medicine 2014 12:303.

\section{Submit your next manuscript to BioMed Central and take full advantage of:}

- Convenient online submission

- Thorough peer review

- No space constraints or color figure charges

- Immediate publication on acceptance

- Inclusion in PubMed, CAS, Scopus and Google Scholar

- Research which is freely available for redistribution 